\title{
Chlorpromazine, placebo and droperidol in the treatment of nausea and vomiting
} associated with cisplatin therapy

\author{
R. STUART-HARRIS \\ M.B., B.S., M.R.C.P. \\ R. BUCKMAN \\ M.B., B.Chir., M.R.C.P. \\ I. STARKE \\ M.B., B.S., M.R.C.P. \\ EVE WILTSHAW \\ M.D., F.R.C.P. \\ Department of Medicine, Royal Marsden Hospital, Fulham Road, London SW3
}

\section{Summary}

The use of cisplatin may be associated with severe nausea and vomiting. Two separate, randomized, double-blind trials, comparing the anti-emetic effect of chlorpromazine with placebo and chlorpromazine with droperidol, were conducted in patients receiving cisplatin for ovarian cancer. Chlorpromazine was statistically superior to placebo in the control of nausea and vomiting in those patients receiving lowdose cisplatin therapy. Patients treated with chlorpromazine had significantly less nausea than with droperidol, but there were no other significant differences between chlorpromazine and droperidol. Toxicities of chlorpromazine and droperidol were similar. Chlorpromazine shows useful activity against cisplatin nausea and vomiting.

KEY WORDS: chlorpromazine, placebo, droperidol, cisplatin, nausea, vomiting.

\section{Introduction}

Nausea and vomiting are common side effects of many chemotherapeutic agents used in the treatment of malignant disease (Seigel and Longo, 1981). The control of chemotherapy-associated nausea and vomiting is important as these adverse effects may prejudice patients from receiving chemotherapy for potentially curable tumours. Cisplatin, an agent used in the treatment of ovarian cancer, may cause severe nausea with continuous vomiting (Jacobs, Deppe and Cohen, 1980). Chlorpromazine has established antiemetic activity although there has been no previous double-blind trial of chlorpromazine $v$. placebo in the treatment of chemotherapy-induced nausea and vomiting. We have compared chlorpromazine with placebo and subsequently carried out the first double-blind trial of chlorpromazine $v$. droperidol, an agent reported to have useful anti-emetic activity against cisplatin-induced nausea and vomiting (Grossman, Lessin and Cohen, 1979).

\section{Materials and methods}

Two consecutive, randomized, double-blind tria $\vec{\downarrow}$ were completed in patients receiving cisplatin alone or in combination for histologically proven ovariaf cancer. Informed consent was obtained from all patients.

\section{Comparison of chlorpromazine and saline}

Twenty consecutive patients were entered into th: trial which compared the use of chlorpromazine with normal saline as placebo. For their ovarian cance\$ patients were treated with high dose cisplatin 100 $\mathrm{mg} / \mathrm{m}^{2}$ by intravenous bolus ( 7 patients), or low dose cisplatin $20 \mathrm{mg} / \mathrm{m}^{2}$ by i.v. bolus in combination with chlorambucil, or hydroxymethylemelamine, or chle rambucil and adriamycin (13 patients).

Chlorpromazine was given in a dose of $40 \mathrm{mg} / \mathrm{m}^{2}$. by intramuscular injection at the start of chem therapy treatment. It was subsequently administered in a dose of $20 \mathrm{mg} / \mathrm{m}^{2}$ i.m. 4 hourly for $12 \mathrm{hr}$ and the $20 \mathrm{mg} / \mathrm{m}^{2}$ i.m. 4 hourly on request for the rest of the admission. Normal saline was given in identical viałs and at the same volume and times as chlorpromazine

Patients were randomized to receive chlorprom zine or placebo for separate courses according to the digits of their date of birth. For those digits which were even numbers, patients received chlorproms zine whilst for odd numbers, patients received placebo. Thus, a patient with a birth date of 29.03.32 would have received the following sequence of antsemetics: chlorpromazine for courses 1,3 , and $\%$ placebo for courses 2,4 , and 5 .

Nursing staff recorded episodes of vomiting, natio sea, and drowsiness, the blood pressure and pulse, 
and any other symptoms. After their second and subsequent courses of chemotherapy patients were interviewed by an independent observer and asked to compare their current chemotherapy course with their previous one by means of a numerical scale of preference. Patients were questioned about: (1) overall assessment of the chemotherapy course; (2) appetite; (3) nausea; (4) vomiting; (5) drowsiness; (6) whether they liked to be drowsy; and (7) other side effects encountered.

In both trials, the recorded episodes of vomiting during each treatment period were analyzed using the Generalized Linear Interactive Model (G.L.I.M.) system (Baker and Nelder, 1978).

\section{Comparison of chlorpromazine and droperidol}

Sixteen consecutive patients were entered into this trial which compared the use of chlorpromazine with droperidol. The patients were treated either with high dose cisplatin $100 \mathrm{mg} / \mathrm{m}^{2}$ by single i.v. bolus or in divided doses over 4 days (10 patients) or low dose cisplatin $20 \mathrm{mg} / \mathrm{m}^{2}$ by i.v. bolus (6 patients).

Chlorpromazine $40 \mathrm{mg} / \mathrm{m}^{2}$ by i.v. bolus was given at the start of the chemotherapy treatment. This was followed $4 \mathrm{hr}$ later by a dose of $20 \mathrm{mg} / \mathrm{m}^{2}$ intramuscularly. A similar intramuscular dose was then given 4 hourly on request. For those patients receiving chemotherapy over 4 days, chlorpromazine was given in a dose of $20 \mathrm{mg} / \mathrm{m}^{2}$ i.v. with chemotherapy on days 2,3 and 4 and then 4 hourly intramuscularly as required. Droperidol was administered in a similar manner in a dose of $4 \mathrm{mg} / \mathrm{m}^{2}$ initially and $2 \mathrm{mg} / \mathrm{m}^{2}$ subsequently. Randomization, assessment of nausea and vomiting, and statistical analysis were all carried out as in the first trial of chlorpromazine and saline.

\section{Results}

\section{Comparison of chlorpromazine and saline}

Objective assessment. Twenty patients completed 63 courses of chemotherapy during the study, enabling 43 comparisons of anti-emetic treatment to be made. There were 25 comparisons between chlorpromazine and placebo (10 of chlorpromazine followed by placebo, and 15 of placebo followed by chlorpromazine). The episodes of vomiting during these comparisons are shown in Fig. 1.

Using the statistical model, chlorpromazine was found to be superior to placebo in reducing the number of episodes of vomiting during chemotherapy $(P<0.05)$.

Data from patients receiving their first course of chemotherapy in the study were also compared. Twelve patients received placebo and 8 received chlorpromazine. Vomiting was significantly less in
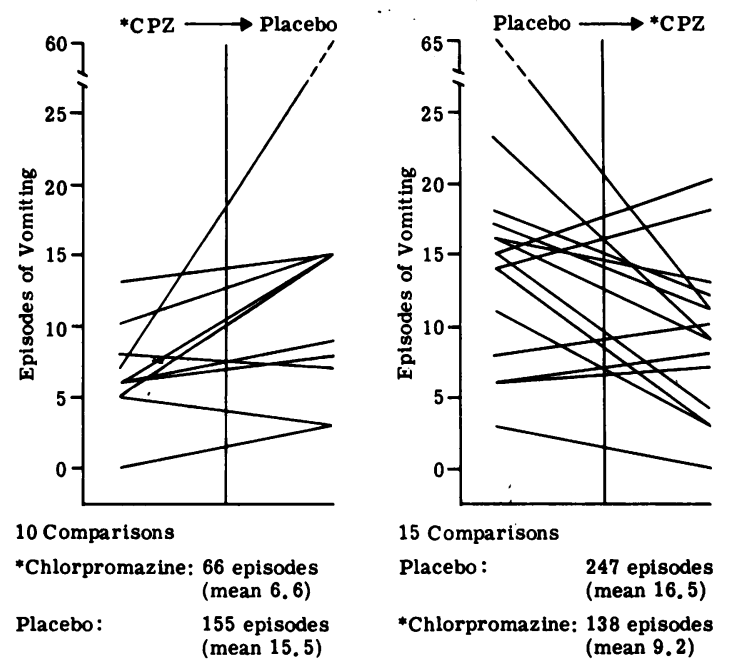

FIG. 1. Episodes of vomiting during comparisons between chlorpromazine and placebo.

the patients receiving chlorpromazine $(P<0.01 t$-test, $P<0.05$ Mann-Whitney U test).

To test whether chlorpromazine was effective in those patients receiving high dose cisplatin and those receiving low dose cisplatin, the patient groups were separated and the statistical model re-applied. This analysis showed that chlorpromazine was superior to placebo only in those patients receiving the low-dose regimen $(P<0.01)$.

Patients' subjective assessment. A summary of the results of the questionnaires is shown in Table 1. Of the 25 comparisons between chlorpromazine and placebo, chemotherapy with chlorpromazine was preferred in 21 instances, placebo preferred in one instance and no preference shown in 3 instances.

Data from patients receiving the first 2 courses of chemotherapy were also analyzed. An ordinary sign test was applied to these results. Chemotherapy with chlorpromazine was preferred by patients $(P<0.002)$ who noted improvement in appetite $(P<0.030)$, nausea $(P<0.001)$, and vomiting $(P<0.004)$. However, treatment with chlorpromazine was associated with drowsiness $(P<0.022)$ whereas treatment with placebo did not produce drowsiness.

There were no statistically significant differences found during this trial when comparing chlorpromazine with chlorpromazine, or placebo with placebo.

Side effects. Drowsiness was the most common side effect noted by patients during chlorpromazine therapy. Dry mouth was also experienced by 6 patients receiving chlorpromazine, but no extra- 
TABLE 1. Patients' subjective preferences for anti-emetic during trials 1 and 2

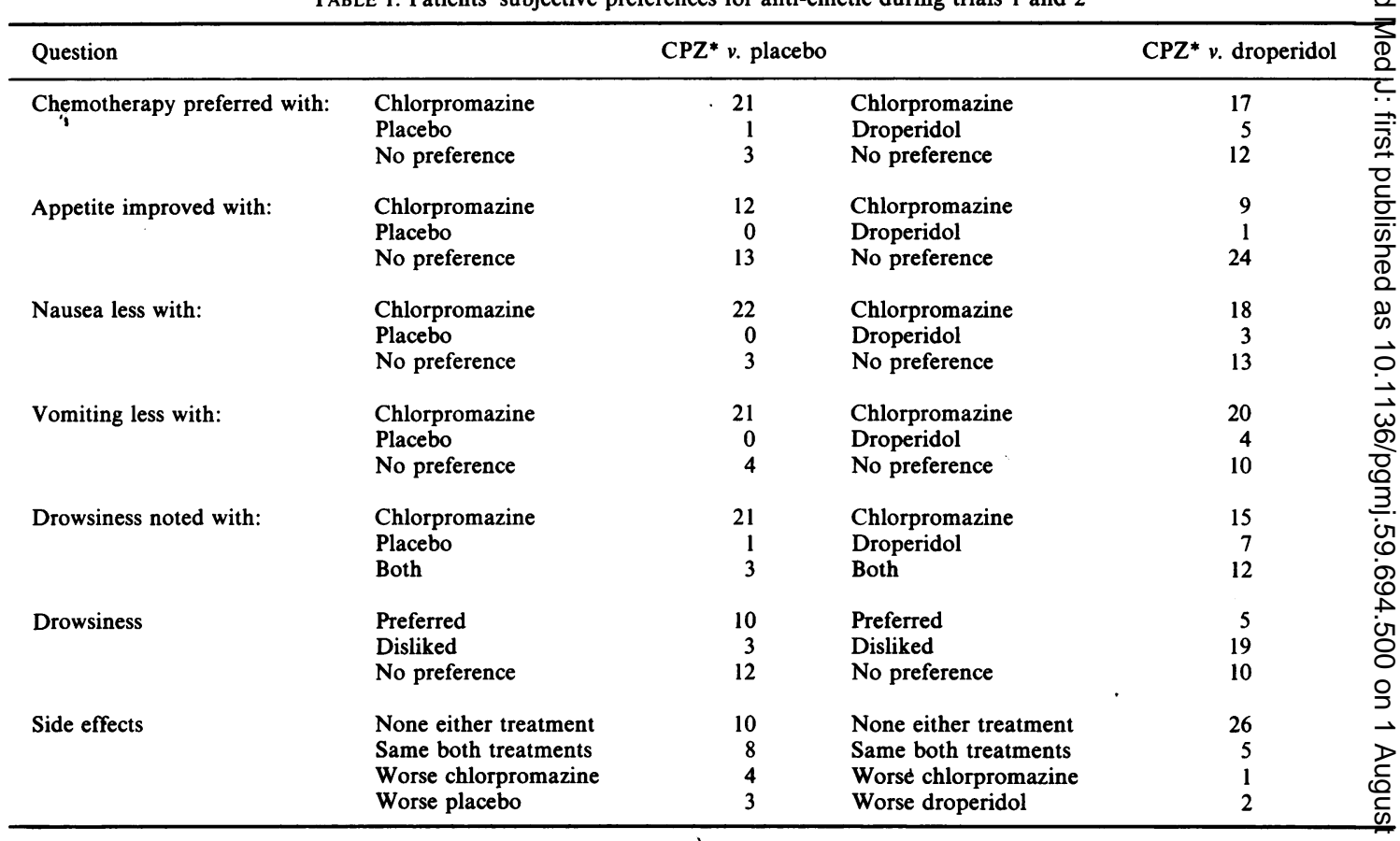

*Chlorpromazine.

pyramidal reactions or hypotensive episodes were observed.

\section{Comparison of chlorpromazine and droperidol}

Sixteen patients completed 70 courses of chemotherapy during the study, enabling 54 comparisons of anti-emetic therapy to be made. There was a total of 34 comparisons between chlorpromazine and droperidol (18 of chlorpromazine followed by droperidol and 16 of droperidol followed by chlorpromazine). Episodes of vomiting during these comparisons are shown in Fig. 2.

Objective assessment. Using the statistical model, there was no significant difference between chlorpromazine and droperidol in the control of vomiting during this study.

Data from patients receiving their first course of chemotherapy in this study were also compared. Eleven patients received chlorpromazine, and 5 received droperidol. There was no statistically significant difference in the incidence of vomiting between the 2 groups ( $t$-test, Mann-Whitney U test).

Patients receiving high-dose cisplatin and those receiving low-dose regimens were compared as in the first trial. There was no statistically significant difference between treatment with chlorpromazine or droperidol.

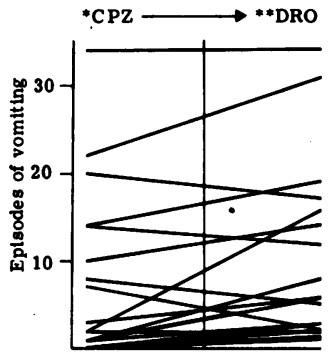

18 Comparisons

*Chlorpromazine: 139 episodes

(mean 7.7)

$\begin{array}{ll}\text { **Droperidol: } & 183 \text { episodes } \\ \text { (mean 10.2) }\end{array}$

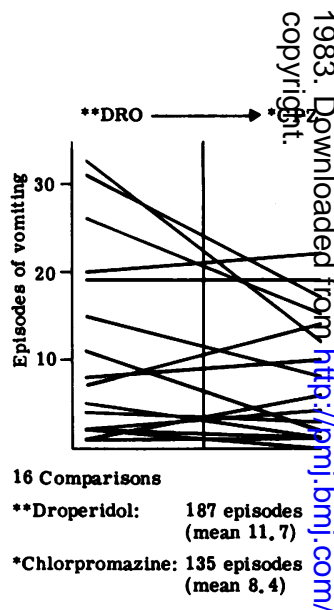

FIG. 2. Episodes of vomiting during comparisons between chlorp용mazine and droperidol.

Patients' subjective assessment. A summary of the patient preferences is shown in Table 1. Of the 34 comparisons of chlorpromazine to droperidds chemotherapy with chlorpromazine was preferred 17 , droperidol in 5 , and no preference shown in 125

Preferences for the first 2 courses of therapy were also compared. An ordinary sign test was applied these results. When comparing chlorpromazine droperidol there were no statistically significant differences for chemotherapy preference, appetite, 
vomiting, or drowsiness. Nausea, however, was significantly less with chlorpromazine $(P<0.032)$.

There were no significant differences when comparing chlorpromazine with chlorpromazine or droperidol with droperidol.

Side effects. Drowsiness was the most frequent side effect and occurred during chlorpromazine and droperidol treatment. One patient experienced diarrhoea during both anti-emetic treatments, and another also complained of restlessness of the legs during both treatments. One patient experienced headache during chlorpromazine treatment.

\section{Discussion}

Although chlorpromazine and droperidol are both believed to act at the level of the chemoreceptor trigger zone in the floor of the fourth ventricle, they belong to different chemical classes. Chlorpromazine is a phenothiazine, droperidol a butyrophenone. Phenothiazines have been thought to have limited application in the treatment of vomiting caused by powerful emetic agents such as cisplatin. Droperidol, however, has been reported to have good activity against cisplatin nausea and vomiting when used alone (Wilson et al., 1981), or in combination with benzquinamide (Paladine et al., 1980).

Droperidol has previously been compared with another phenothiazine, prochlorperazine, in previous studies. In one study, droperidol was compared with prochlorperazine alone in the treatment of cisplatin vomiting. Droperidol treatment was associated with a significantly shorter duration of vomiting (Jacobs $e t$ al., 1980). In another study, droperidol was compared to prochlorperazine in combination with trimethobenzamide and was found to be somewhat more effective despite lack of statistical significance (Mehrotra et al., 1981).

The results of the first part of our study indicate chlorpromazine is superior to placebo in reducing vomiting in patients on low-dose cisplatin but not in those on high-dose therapy. This may reflect the smaller number of patients in the high-dose group having comparisons between chlorpromazine and placebo rather than a failure of chlorpromazine. The results of the second part of the trial show that there was no significant difference between chlorproma- zine and droperidol in the control of vomiting, but that nausea was significantly less with chlorpromazine treatment. More patients preferred chlorpromazine than droperidol, but this preference was not significant.

With the statistical model that was employed, there was a significant difference between different patients, and also different treatment periods. These effects may have come about because one patient had infrequent vomiting during one course, followed by very severe vomiting on the subsequent course, despite the administration of the same anti-emetic on both occasions. The side effects of chlorpromazine and droperidol were similar, despite the higher dose of droperidol used compared to previous studies.

In conclusion, chlorpromazine may show useful activity against cisplatin-induced nausea and vomiting, but we were unable to confirm previous reports that droperidol is particularly effective.

\section{Acknowledgments}

We wish to acknowledge the assistance and co-operation received from all the nursing staff involved during the course of these studies.

\section{References}

BAKER, R.J. \& NeLDER, J.A. (1978) The GLIM System-Manual N.A.G., Oxford.

Grossman, E., Lessin, L.S. \& Cohen, P. (1979) Droperidol prevents nausea and vomiting from cis-platinum. New England Journal of Medicine, 301, 47.

JACOBS, A.J., DEPPE, G. \& COHEN, C.J. (1980) A comparison of the anti-emetic effects of droperidol and prochlorperazine in chemotherapy with cis-platinum. Gynaecologic Oncology, 10, 55.

Mehrotra, S., Rosenthal, C.J., Barile, B., Dotan, S. \& AlFONSO, A. (1981) A comparison between droperidol (DP) and prochlorperazine (PCP) in combination with trimethobenzamide (TMB) as anti-emetic for antinoplastic combination chemotherapy (CCT). Proceedings of the American Association of Cancer Research and American Society of Clinical Oncology, 22, 417.

Paladine, W., Price, L., Sokol, G., Kritz, E. \& Ayres, V. (1980) Antiemetic trial of droperidol. Proceedings of the American Association of Cancer Research and American Society of Clinical Oncology, 21, 380.

SIEGEL, L.J., \& LONGO, D.L. (1981) The control of chemotherapyinduced emesis. Annals of Internal Medicine, 95, 352.

Wilson, J., Weltz, M., Solimando, D., Perry, D., Baldwin, P. \& KimBall, D. (1981) Continuous infusion droperidol: anti-emetic therapy for cis-platinum (DPP) toxicity. Proceedings of the American Association of Cancer Research and American Society of Clinical Oncology, 22, 421.

(Accepted 20 January 1983) 